\title{
扰动环境中不同刏割方式对柠条营养生长补偿的影响
}

\author{
方向文 ${ }^{1}$ 王万鹏 2 何小琴3 ${ }^{2}$ 王 刚 $^{1 *}$ \\ (1 兰州大学干旱与草地农业生态教育部重点实验室 兰州 730000) \\ （2 兰州市南北两山环境绿化工程指挥部，兰州 730046）（3 甘肃农业大学林学院, 兰州 730070)
}

摘 要 柠条 (Caragana korshinskii) 在地上组织破坏后进行补偿性生长, 这是重复利用柠条资源的基础, 但对柠条 不同刚割方式下营养生长补偿的模式有待探讨。该文通过 5 种刚割方式 :去除主枝长的 30\%(30\% RSL)、去除主枝 长的 60\% (60\% RSL)、去除分枝数的 $25 \%$ (25\% RSN)、去除分枝数的 $50 \%$ (50\% RSN) 和去除分枝数的 $100 \%$ ( $100 \% \mathrm{RSN})$ 来研究柠条的营养生长补偿。结果表明 : 刈割处理的柠条生物量当年发生了超补偿, 当年生枝数/枝、 当年生枝长、当年生枝生物量/枝、当年生枝生物量/株比对照高。对照、30\% RSL 和 $60 \%$ RSL 处理未长出基梢。 100\% RSN 处理的基梢数/刚割枝、基梢长、单个基梢平均生物量显著高于 $25 \% \mathrm{RSN}$ 和 $50 \% \mathrm{RSN}$ 处理,基梢生物量/ 株随刈割去除生物量的增加而增加。100\% RSN 处理未结果, 其它处理果实产量表现出超补偿或精确补偿。对照 处理营养生长和生殖生长均低, 其它处理当年生枝生物量与果实产量成显著负相关。从整个生长季节来看, 营养 生长主要集中在果实成熟之前。我们认为, $100 \% \mathrm{RSN}$ 处理是柠条地上组织破坏后尽快恢复的合理方式, 其当年生 生物量远高于其它处理。顶端优势的破坏促使休眠芽的萌发, 根冠比的改变使地上组织获得较多养分和水分, 根 系储存的碳水化合物的供应是促使刏割柠条营养生长超补偿的的可能机制, 而减少生殖生长对资源的消耗, 是 $100 \%$ RSN 处理地上生物量尽快恢复的另一重要因素。

关键词 柠条 当年生枝 基梢 营养生长补偿 邚割

\section{A STUDY ON VEGETATIVE COMPENSATORY GROWTH OF SHRUB , CARAGANA KORSHINSKII , UNDER DIFFERENT CLIPPING TREATMENTS IN DISTURBANCE ENVIRONMENT}

\author{
FANG Xiang-Wen ${ }^{1}$ WANG Wan-Peng ${ }^{2}$ HE Xiao-Qin ${ }^{3}$ and WANG Gang ${ }^{1 *}$ \\ (1 Key Laboratory of Arid and Grassland Agroecology at Lanzhou University, Ministry of Education, Lanzhou 730000 , China) \\ (2 Lanzhou Administration of Environment and Afforestation of South-North Mountains, Lanzhou , 730046 , China) \\ (3 Forestry College of Gansu Agricultural University , Lanzhou , 730070 , China)
}

\begin{abstract}
Background and Aims After browsing or clipping, Caragana korshinskii can compensate for loss of biomass, which is the foundation for repeated use of resources of this species. However, the pattern of vegetative compensatory growth under different clipping treatments is still poorly understood.

Methods In order to test the effects of removal of partial shoot length (RSL) and partial shoot number (RSN) on vegetative growth, we removed 30\% (30\% RSL), 60\% (60\% RSL) of main shoot length, and $25 \%(25 \% \mathrm{RSN}), 50 \%(50 \% \mathrm{RSN})$, and $100 \%$ (100\% RSN) of main shoot number.

Key Results Clipped shrubs overcompensated for tissue loss , and current year shoot number per shoot, current year shoot length, current year shoot biomass per shoot and current year shoot biomass per shrub were higher than those of the control. The control , 30\% RSL and 60\% RSL shrubs did not resprout after clipping , while $25 \% \mathrm{RSN}, 60 \% \mathrm{RSN}$ and $100 \% \mathrm{RSN}$ treatments did resprout and the biomass per shrub increased when more shoot biomass were removed. Differences in resprout number per removal shoot, resprout length , individual resprout biomass were not significant between 25\% RSN and 50\% RSN treatment, but resprout number per removal shoot, resprout length, individual resprout biomass of both were less than those of $100 \%$ RSN treatment. Fruit production in clipping treatments was higher or equal to that of the control with the exception of $100 \%$ RSN , which grew no fruit. There was a negative relationship between annual shoot biomass and fruit production with the exception of the control, which had less fruit production and the annual shoots almost did not grow. During growth season , biomass of current year vegetative growth increased quickly from current year' $\mathrm{s}$ shoot growth to fruit ripeness. In the following three months, from fruit ripeness to leaf abscission , however,
\end{abstract}


it increased slowly.

Conclusions $100 \%$ RSN treatment may be a reasonable mode to prompt shrubs to recover quickly from damage. Plants' responses associated with compensation may include : increased branching or tillering after alteration of the apical dominance , increased availability of water and/or nutrients after the change in the ratio of shoot to root, and remobilization of resources reserved in roots to aboveground organs. Decreased allocation of resources to reproductive growth may be another important mechanism for $100 \%$ RSN shrubs to compensate for the loss of organs.

Key words Caragana korshinskii , Current year shoot, Resprout , Vegetative compensatory growth , Clipping

由于人们的采伐利用或动物的采食, 木本植物 地上部分经常遭受破坏。在陆地生态系统扰动的环 境中, 许多植物在破坏后会进行补偿性生长( Bond \& Midgley ,2001; Cruz et al. ,2003)。Belsky(1986) 将 补偿分为超补偿、精确补偿和不足补偿, 并分别定 义为植物的累积干重 (包括修剪或采食的生物量)大 于、等于或小于对照植物。植物的补偿能力通常取 决于物种、采食或刈割的强度、方式和时间 (Maschinski \& Whitham , 1989 ; Simons \& Johnston , 1999)。

萌蒒植物根系或根状茎具有大量的休眠芽 (Bowen \& Pate , 1993 ;Cruz \& Moreno，2001) ,储存着 大量的碳水化合物和矿物营养, 在地上组织破坏后 具有较强的生长补偿能力。Pate 等 (1990)认为萌葟 植物通常生长速率较慢, 有较高的根冠比, 根系储存 大量的淀粉。在地上部分受到破坏的早期，根系储 存的碳水化合物将运到地上, 供应地上部分的生长 (Bowen \& Pate , 1993 ; van der Heyden \& Stock , 1996 ; Canadell \& López-Soria , 1998), 同时伴随着较高的叶 片光合速率, 使地上生物量快速恢复 (Fleck et al., 1998)。

柠条 (Caragana korshinskii) 是生长在干旱和半干 旱地区典型的萌蒒植物, 其根系发达, 主根可深达 5 10 m (王全珍等, 2001)。枝条萌菜力和再生能 力极强, 特别是经过平茬以后, 能从根颈部萌生出大 量枝条, 形成稠密的灌丛。研究表明柠条在一年中 尽管春夏秋冬牲畜多次啃食枝叶，生长仍然十分旺 盛, 充分显示了其极强的再生能力。柠条不仅可以 放牧 将其枝条加工成草粉饲喂畜禽, 也是很有发展 潜力的天然绿色饲料资源，已有不少专著和期刊论 述和介绍(刘国谦等 2003)。柠条还可以做为燃料, 解决农村的能源问题(王孟本等,1996)。

根据已有文献和柠条生物学特性, 我们推测柠 条在地上部分被破坏后会产生补偿或超补偿, 但在 不同㺫割条件下柠条的营养补偿模式不同。多年来 形成的人工柠条群落的长期围栏封育是对资源的一 种浪费，应当考虑进行合理利用，如割条、樵采等。
本文研究的问题是在不同的则割条件下柠条的补偿 模式有何差异, 采用何种刈割方式可使柠条的生物 量发生最大程度的补偿, 其相关的可能机理如何?

\section{1 材料和方法}

实验地是人工柠条试验田, 位于甘肃省榆中县 北 $90 \sim 100 \mathrm{~km}$ 处。其平均海拔高 $2400 \mathrm{~m}$, 年平均温 度 $6.5{ }^{\circ} \mathrm{C}$ 。年降水量和蒸发量分别为 395 和 1362 $\mathrm{mm}$ (史竹叶等,2003)。

刏割处理在 2004 年冬末春初树液流动之前进 行。选择 60 株均匀分布的 16 年生的柠条作为研究 对象。每株柠条约有 33 个分菜枝, 其冠幅约为 1.5 m。在则割处理前，对每株植物的分枝的基径进行 了测量, 并统计分枝数。实验分 6 个处理 (每个处理 10 株) :自上而下去除主枝长度 (Shoot length removal， RSL)的 30\% (30\% RSL) 和 60\% (60\% RSL) ;去除掉枝 数 (Shoot number removal, RSN) 的 $25 \%(25 \%$ RSN)、 $50 \%$ (50\% RSN) 和 100\% (100\% RSN) ;对照 (未经处 理)。在对枝长度去除处理中 (RSL) 将每株柠条中 每个主枝的中上部分去除，以刺激主干休眠芽萌发 ; 在枝数去除处理中 将植株中 $25 \% 、 50 \%$ 和 $100 \%$ 主 枝数从高于地表 $1 \mathrm{~cm}$ 位置去除, 剩余枝不处理, 并 留以后观测用。为了估测植物地上生物量, 建立了 枝的基径和生物量的方程 $\left(R^{2}=0.92, p<0.001\right)$ ， $30 \%$ RSL、60\% RSL、 $25 \%$ RSN 、 $50 \%$ RSN 和 $100 \%$ RSN 处理分别对应去除植株地上生物量的 $17 \% 、 55 \%$ 、 $20 \% 、 45 \%$ 和 $100 \%$ 。实验柠条在生长期用护栏围 住，以防动物啃食枝叶。

果实成熟时 (8月 2 日) , 采摘每个处理各株柠 条的果实, 分别在烘箱中 $70{ }^{\circ} \mathrm{C}$ 烘 $48 \mathrm{~h}$ 后称重。在 抽稍期 (6月 2 日)、果实发育期 (7月 15 日)、果实成 熟期 (8 月 15 日) 和落叶期 (11月 2 日) 统计每株植 物当年生枝数和基梢数目、长度 建立枝长与生物量 之间的回归关系 (Riba，1998) :

$\log W=1.5498 \log L-2.416\left(R^{2}=0.942\right)$

其中 $W$ 为生物量 $(\mathrm{g}) 、 L$ 为枝长 $(\mathrm{cm})$ 统计出当 
年生枝数/枝、当年生枝长 $(\mathrm{cm})$ 、当年生枝生物量/枝 $\left(\mathrm{g}\right.$ 枝 $\left.{ }^{-1}\right)$ 、当年生枝生物量/株 $\left(\mathrm{g}\right.$ 株 ${ }^{-1}$ )、基梢数/刏 割枝、基梢长 $(\mathrm{cm})$ 、单个基梢生物量 $\left(\mathrm{g}\right.$ 基梢 ${ }^{-1}$ )、基梢 生物量/株 $(\mathrm{g}$ 株 -1$)$; 并将每株各指标的均值用 OneWay ANOVA 进行统计分析。对老龄枝条长出的一年 生枝我们称作当年生枝 基部从根颈处长出的一年生 枝称作基梢。本文营养生长仅包括当年生枝生物量 和基梢生物量, 超补偿和精确补偿定义为刏割植物的 营养生长量和果实产量大于或等于对照植物。

\section{2 实验结果}

\section{1 不同处理对当年生枝生长的影响}

由图 1 可知，老年柠条在刚割处理后产生更多 的分枝，总体趋势为 :RSL 处理大于 RSN 处理，大于 对照。则割处理与对照相比差异极显著 $(p<$ $0.001) 。 60 \%$ RSL 分枝数最多，约是对照的 4 倍。 当年生枝长在各处理之间与当年生枝数/枝有相似 的变化趋势，刏割显著促进当年生枝的伸长生长，导 致各刈割处理与对照之间差异极显著 $(p<0.001)$ 。 当年生枝生物量/枝与当年生枝数/枝和当年生枝长 成显著正相关 (分别为 $R^{2}=0.636 、 p=0.011$ 和 $R^{2}$ $=0.937 、 p=0.007 ）$, 使得刈割处理的当年生枝生物 量/枝远高于对照, 尤其是 60\% RSL 处理, 其值是对 照的 42 倍。其它处理 (30\% RSL、25\% RSN 和 50\% RSN) 相对 $60 \%$ RSL 处理低, 差异极显著 $(p<$ 0.001 )。尽管对照植株枝末经刈割，和其它处理相 比有较高的分枝数, 但对每株当年生生物量贡献不 明显，对照植株当年生生物量仍极低。对照植株当 年生长很弱, 几乎停止生长。结果表明 柠条在地上 组织破坏后进行了快速的补偿性生长, 并为超补偿。

\section{2 不同处理对基梢生长的影响}

由图 2 可知，对照、30\% RSL 和 60\% RSL 处理基 部无新梢生长, 说明只有主枝地上部分完全去除，才 能促进根颈休眠芽萌发。100\% RSN 的基梢数/刏割 枝、基梢长、单个基梢生物量高于 $25 \% \mathrm{RSN}$ 和 $50 \%$ RSN 处理。方差分析表明各指标在 $100 \%$ RSN 处理 与 $25 \% \mathrm{RSN}$ 和 $50 \% \mathrm{RSN}$ 处理之间差异极显著 $(p<$ 0.001 ）,但在 $25 \% \mathrm{RSN}$ 与 $50 \% \mathrm{RSN}$ 处理之间基梢数 刏割枝、基梢长、每基梢生物量无显著差异。说明 植物老枝新梢生长和果实的发育抑制了基梢的生 长，它们之间在养分和光合同化产物的分配上存在 竞争。基梢生物量/株与地上部分生物量的刈割强 度有关 强度越大, 基梢生物量越多。

2.3 不同处理对植株不同时间营养生长生物量的
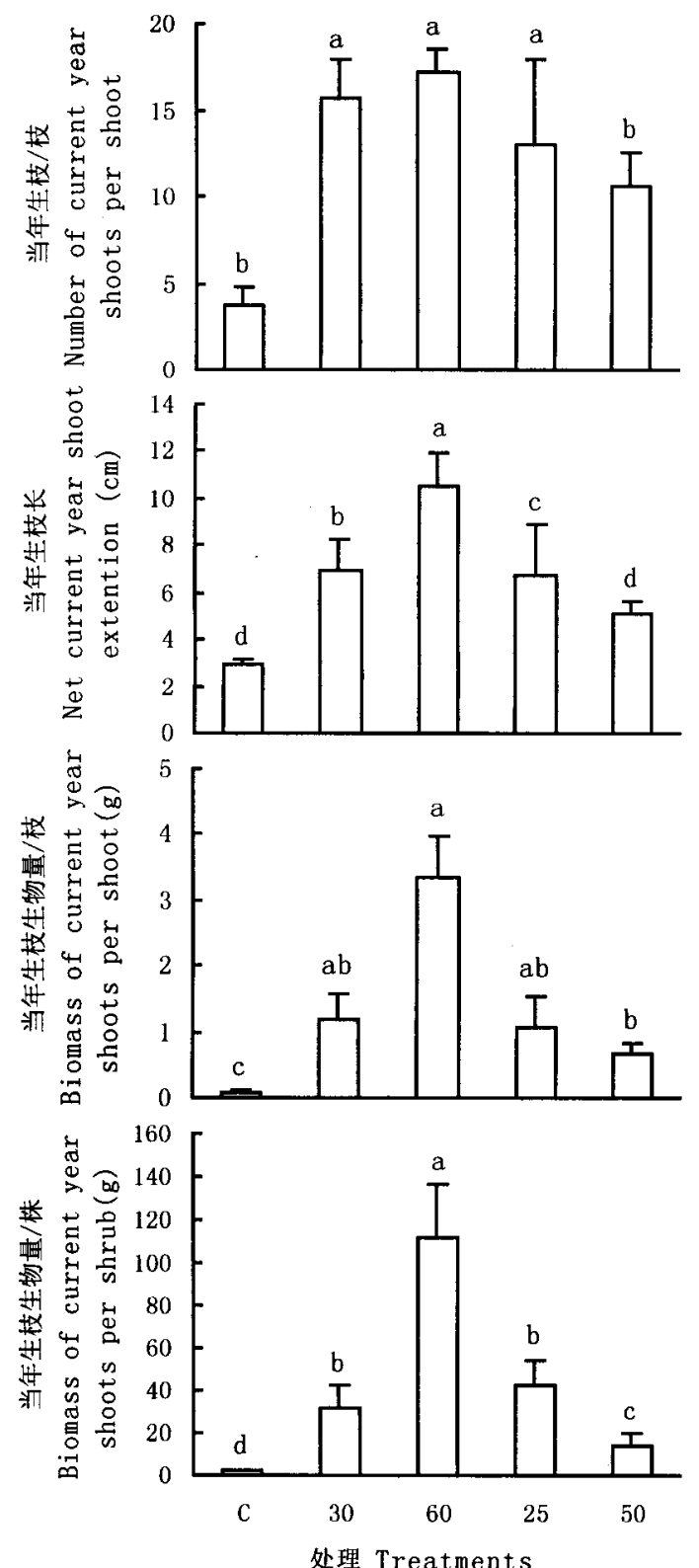

图 1 不同刚割处理对柠条当年生枝数/枝、当年生枝长、当年生 枝生物量/枝、当年生枝生物量/株的影响 $(n=10)$

Fig. 1 The effects of different clipping treatments on current year shoot number per shoot, current year shoot length , current year shoot

biomass per shoot and current year shoot biomass per shrub

C 对照 Control $30: 30 \%$ RSL 60 :60\% RSL 25 :25\% RSN $50: 50 \%$ RSN RSL :去除主枝长度 Shoot length remonal RSN : 去除 掉枝数 Shoot number removal

影响

由图 3 可知 植株在 6 月初开始抽稍 到 8 月之 间生长迅速 ; 之后生长缓慢 到落叶时停止生长。从 图 3 还看出, 营养生长生物量主要取决果实成熟之 前的生长，其生物量约占总生物量的 $80 \%$ 。在果后 营养生长期间 柠条可能将大量的光合产物分配到 根系,并以淀粉的形式存储，以防地上部分再次被采 食或破坏。这可能是柠条对外界扰动环境长期适应 

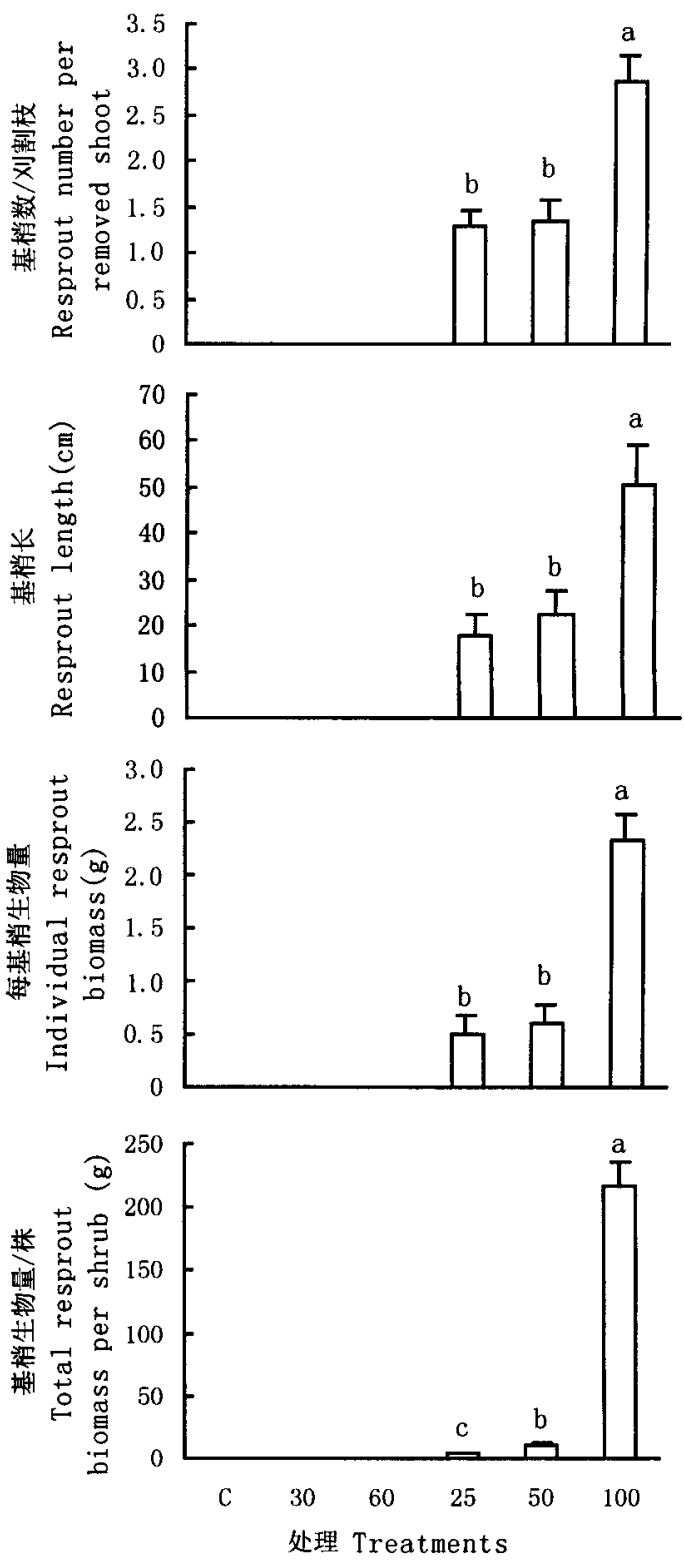

图 2 不同刚割处理对柠条基梢数/刏割枝、基梢长、 单个基梢生物量和基梢生物量/株的影响

Fig. 2 The effects of different clipping treatments on resprout number per removed shoot, resprout length , individual resprout biomass and resprout biomass per shrub

$100: 100 \%$ RSN C、30、60、25、50、RSL、RSN :同图 1 See Fig.1

的结果。

\section{4 不同处理对果实产量的影响}

图 4 表明,30\% RSL、25\% RSN 和 50\% RSN 处理 果实产量远高于对照植株，约 2 倍之多，即为超补 偿。60\%RSL 处理低于对照，但分析表明它们之间 差异不显著, 即精确补偿。100\% RSN 处理当年尚未 开花, 无果实产量。由于果实成熟之前当年生生物 量占总生物量 $80 \%$,因此我们试图建立营养生长与 生殖生长的关系。发现除对照处理外, 各处理营养
生长生物量与果实产量成显著负相关 : $\log$ (Fruit) = $-0.3704 \log ($ Shoot $)+2.5663\left(R^{2}=0.38, p<\right.$ $0.001 ）$,说明果实生长和发育消耗了大量的碳水化 合物和养分 致使营养生长减少。

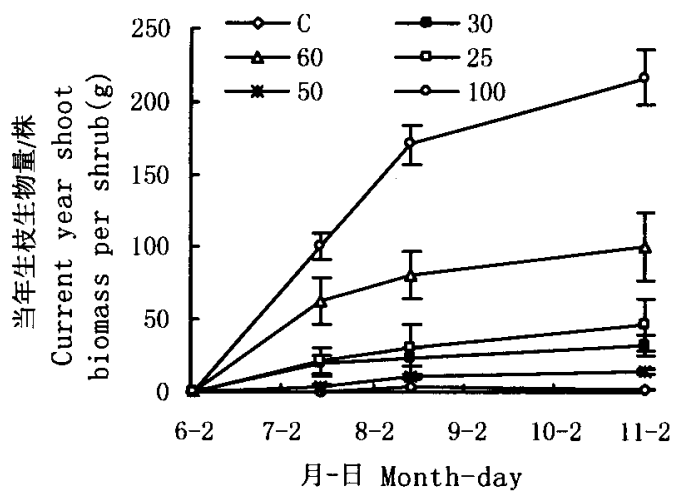

图 3 不同刈割处理对植株不同时间当年生枝生物量的影响

Fig.3 The effects of different clipping treatments on current year shoot biomass at different times

C、30、60、25、50、RSL、RSN : 同图 1 See Fig. 1100 : 同图 2 See Fig. 2

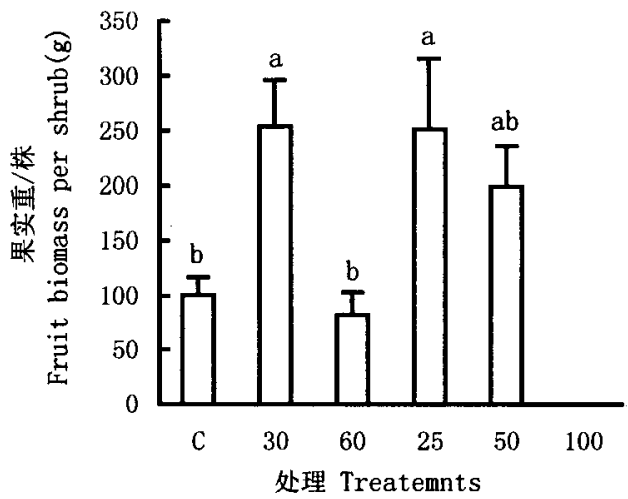

图 4 不同刏割处理对柠条果实产量的影响

Fig. 4 The effects of different clipping treatments on fruit production

C、30、60、25、50、RSL、RSN : 同图 1 See Fig. 1100 : 同图 2 See Fig. 2

\section{3 讨 论}

木本植物通常具有忍耐外界采食和破坏的能力 (Bilbrough \& Richards，1993)，植物在受到生物和非 生物破坏时这种能力使地上生物量快速恢复 (Salemaa et al ，1999)。植物补偿能力取决于众多因素， 包括可利用资源、生物量破坏时间 (Maschinski \& Whitham ,1989 ; Obeso , 1993 ; Trumble et al. , 1993)、 生物量破坏之前碳水化合物储存水平 (Hockwender et al. 2000)、破坏之后植物的光合速率 (Dyer et al.,1991 ; Houle \& Simard , 1996 ; Meyer , 1998)、对 有限资源的分配方式 (Mabry \& Wayne，1997)、顶端 
优势解除 (Escarré et al. , 1996 ; Sacchi \& Connor , 1999 ; Simons \& Johnston, 1999 ; Lortie \& Aarssen , 2000)、枝条之间对养分和水分的竞争减少 (Du Toit et al. , 1990)。柠条地上部分被破坏后存在超补偿， 使得柠条地上部分生物量得以快速恢复, 这是柠条 的复壮更新，再度合理利用的基础。

生长素(Auxin) , 通常由顶端分生组织和刚展开 的幼叶产生, 具有抑制侧芽生长发育功能, 并促使 养分供应已有的分生组织(Phillips,1975)。细胞分 裂素 (Cytokinins) 能促进细胞分裂和侧芽萌发 (Bangerth et al ，2000), 并能有效地调动养分 (Fetene \& Beck，1993)。当地上分生组织破坏后, 生 长素的形成将被阻断, 导致生长素和细胞分裂素之 比减少和大量的侧芽萌发 (Phillips, 1975 ; Simons \& Johnston , 1999 ; Lortie \& Aarssen , 2000) ,从而使得植 物继续生长。比如, 移去新分藍枝导致大量分菜枝 萌发和生长 (Schier , 1972 ; Frey et al .,2003)。随克 隆植物分株数移去的增多, 萌菜枝的生物量增加 (Riba，1998)。在 30\% RSL 和 60\% RSL 处理中，主枝 的顶端分生组织全部去除，而 $25 \%$ RSN 和 $50 \%$ RSN 处理中只有 $25 \%$ 和 50\%的分生组织去除，导致 30\% $\mathrm{RSL}$ 和 $60 \% \mathrm{RSL}$ 处理的当年生枝数/枝比 $25 \% \mathrm{RSN}$ 和 $50 \% \mathrm{RSN}$ 处理高, $100 \% \mathrm{RSN}$ 处理的基梢数/刘割 枝比 $25 \%$ RSN 和 $50 \%$ RSN 处理高。我们的研究同 样支持上述结论, 并说明柠条产生大量一年生枝来 恢复地上生物量。

除对照植株外, 其它处理营养生长生物量与果 实产量成负相关, 表明生殖生长和营养生长之间存 在对养分的竞争, 以前的研究结果同样表明这一点

(Sutherland \& Vickery , 1988 ; Reekie，1991；Westley , 1993)。植物资源的分配方式是植物在进化过程中 在生长、资源储存、生殖和防御之间长期权衡的结果 (Cruz \& Moreno，2001)。植物生殖生长需要大量能 量, 导致对其它器官能量分配的减少。如随着植物 对有性生殖能量分配的提高, 对无性繁殖器官(如根 状茎) 分配的能量会减少 (Sutherland \& Vickery, 1988 ; Reekie，1991；Westley，1993)。柠条刚割后， 光合产物将在营养生长和生殖生长之间进行权衡, 从提高当年适合度而言, 应减少营养生长; 从柠条地 上部分生物量利用而言, 应减少生殖生长。我们的 研究表明, $100 \%$ RSN 处理当年末开花结果, 有着较 高的当年生生物量。对以薪炭林为用途的柠条来 说, $100 \% \mathrm{RSN}$ 处理是较为合理的一种方式。

和其它灌木类似(Landhäusser \& Lieffers，2002)，
柠条根系储存的碳水化合物随生长季节而变化, 早 春花蕾发育和展叶时, 根系将大量的储存物质供应 地上部分生长, 储存物质含量达最低值。随后其功 能叶的光合产物向地下运输，以淀粉形式存储 秋末 落叶后根系储存物质含量达最高, 冬季随根呼吸作 用而略有降低(Fang et al. , 2006)。故若利用柠条 地上部分生物量，刘割应在秋末到春初树液流动以 前进行为好。有大量的研究证明与我们的这一观点 相同 (Maschinski \& Whitham , 1989；Obeso , 1993 ; Trumble et al. , 1993)。如熊果 (Arctostaphylos uva-ur$s i$ )和笃斯 (Vaccinium uliginosum) 在秋季修剪后有较 高的补偿能力, 而春季修剪后补偿能力较低 (Salemaa et al. ,1999)。

许多植物在自然条件或人为因素下地上组织被 破坏, 如地中海地区的森林火灾经常破坏当地植物 的地上部分 (Bond \& Midgley, 2001; Cruz et al. , 2003 ）,使植株重新长出地上组织。原有强大的根系 储存的营养 (Fleck et al. , 2000)、吸收的水分 (Wellington，1984; Saruwatari \& Davis ,1989)将供给有 限的地上部分, 因此萌菜枝在第一年时其生长速率、 气体交换等许多方面都较高。在干旱半干旱地区， 植物常年受到干旱的胁迫, 水分成为这一地区植物 生长的限制因子(Al-Khayri \& Al-Bahrany , 2004)。有 研究表明, 破坏植物的地上组织后, 植被周围土壤储 水因植物蒸腾的减少而暂时提高, 这将改善萌菜枝 的供水状况; 汢壤水分随萌蒒萻枝生长而逐渐减少，但 回到原来水平也需要几年的时间 (Wellington,1984)。 柠条是一种广泛分布于我国西北干旱半干旱地区的 灌木, 当地上部分破坏后, 导致根冠比严重失调, 地 上枝条之间的竞争减小 (du Toit，1990) ,使得有限的 地上部分能够获得较多的养分和水分，导致扰动破 坏后第一年叶片有较高的光合速率, 使得枝条生长 迅速 (Fang et al. , 2006)。有大量的研究得出同样 的结论 (Saruwatari \& Davis, 1989 ; Kruger \& Reich , 1997 ; Fleck et al ., 1998)。

尽管柠条在刚割后第一年生长迅速，但地上生 物量恢复到原来的水平需要几年的时间，未来几年 中刈割的柠条受养分和水分胁迫和对照相比仍较 小, 营养生长仍为超补偿。但是, 这种补偿能力会呈 指数式迅速下降。一旦根冠比达到对照植物所处的 平衡状态, 营养生长不再发生补偿。

Haukioja 和 Koricheva (2000) 认为多年生木本植 物树冠通常表现出老龄化症状，枝条、小枝甚至主干 开始干枯、死亡, 修剪树干和枝条是促使树木幼龄化 
和旺盛生长的必须措施。幼龄化的植株产生大量具 有较大生物量的长枝, 是产生补偿的基础。本文也 证明刏割是促使老年柠条复壮更新的有效措施。

本文研究认为, 刏割使老年柠条复壮更新, $100 \%$ RSN 刏割处理是促进柠条地上生物量快速恢 复的有效利用方式。柠条作为干旱、半干旱地区一 种重要的燃料资源, 刏割时应充分考虑剽的方式 和植物的补偿和超补偿效应, 对可再生资源进行合 理的利用。

\section{参 考 文 献}

Al-Khayri JM, Al-Bahrany AM (2004). Growth, water content, and proline accumulation in drought-stress callus of date palm. Biologia Plantarum, 48, $105-108$.

Bangerth F, Li CJ, Gruber J (2000). Mutual interaction of auxin and cytokinins in regulating correlative dominance. Plant Growth Regulation, 32, $205-217$.

Belsky AJ (1986) . Does herbivory benefit plants? A review of the evidence. American Naturalist, 127, 870-892 .

Bilbrough CJ, Richards JH (1993) . Growth of sagebrush and bitterbrush following simulated winter browsing: mechanisms of tolerance. Ecology, 74, 481-492.

Bond WJ, Midgley JJ (2001) . Ecology of sprouting in wood plants: the persistence niche. Trends in Ecology and Evolution, 16, 45 -51 .

Bowen BJ, Pate JS (1993). The significance of root starch in postfire shoot recovery of the resprouter Stirlingia latifolia R. Br. (Proteaceae). Annals of Botany, 72, 7-16.

Canadell J, López-Soria L (1998) . Lignotuber reserves support regrowth following clipping of two Mediterranean shrubs. Functional Ecology, 12, $31-38$.

Cruz A, Moreno JM (2001). No allocation trade-offs between flowering and sprouting in the lignotuberous, Mediterranean shrub Erica australis. Acta Oecologica, 22, $121-127$.

Cruz A, Pérez B, Moreno JM (2003). Plant stored reserves do not drive resprouting of the lignotuberous shrub Erica australis. New Phytologist. 157, $251-261$.

du Toil JT, Bryant JP, Fisby K (1990) . Regrowth and palatability of Acacia shoots following pruning by African savanna browsers. Ecology, 71, 149-154.

Dyer MI, Acra MA, Wang GM, Coleman DC, Freckman DW, McNaughton SJ, Strain BR (1991). Source-sink carbon relations in two Panicum coloratum ecotypes in response to herbivory. Ecology, 72, $1472-1483$.

Escarré JE, Lepart J, Sentuc JJ (1996) . Effects of simulated herbivory in three old field Compositae with different inflorescence architectures. Oecologia, 105, 501-508.

Fang XW, Wang XZ, Li H, Chen K, Wang G (2006). Responses of Caragana korshinskii to different aboveground shoot removal: combining defence and tolerance stragegies. Annals of Botany,
$98,203-211$.

Fetene M, Beck E (1993). Reversal of direction of photosynthate allocation in Urtica dioica L. plants by increasing cytokinin import into the shoot. Botanica Acta, 106, 235-240.

Fleck I, Aranda X, El Omari B, Permanyer J, Abadía A, Hogan KP (2000) . Light energy dissipation in Quercus ilex resprout after fire. Australian Journal of Plant Physiology, 27, 129- 137.

Fleck I, Hogan KP, Lorens L, Abadía A, Aranda X (1998). Photosynthesis and photoprotection in Quercus ilex resprouts after fire. Tree Physiology, 18, 607-614.

Frey BR, Lieffers VJ, Landhäusser SM, Comeau PG, Greenway KJ (2003) . An analysis of sucker regeneration of trembling aspen. Canadian Journal of Forest Research, 33, $1169-1179$.

Haukioja E, Koricheva J (2000). Tolerance to herbivory in woody vs. herbaceous plants. Evolutionary Ecology, 14, $551-562$.

Hockwender CG, Marquis RJ, Stowe KA (2000). The potential for and constraints on the evolution of compensatory ability in Asclepias syriaca. Oecologia, 122, $361-370$.

Houle G, Simard G (1996). Additive effects of genotype, nutrient availability and type of tissue damage on the compensatory response of Salix planifolia ssp. planifolia to simulated herbivory. Oecologia, 107, $373-378$.

Huhta AP, Hellström K, Rautio P, Tuomi J (2003) . Grazing tolerance of Gentianella amarelle and other monocarpic herbs: why is tolerance highest at low damage levels? Plant Ecology, 166, 49 -61 .

Kruger El, Reich PB (1997). Response of hardwood regeneration to fire in mesic forest opening. II . Leaf gas exchange, nitrogen concentration, and water status. Canadian Journal of Forest Research, 27, $1832-1840$.

Landhäusser SM, Lieffers VJ (2002). Leaf area renewal, root retention and carbohydrate reserves in a colonal tree species following above-ground disturbance. Journal of Ecology, 90, 658 665 .

Liu GQ (刘国谦), Zhang JB (张俊宝), Liu DQ (刘东庆) (2003). The technology of Caragana rororshisrii powder processing and feeding. Pratacultural Science (草业科学), 20, 26 32. (in Chinese with English abstract)

Lortie CJ, Aarssen LW (2000). Fitness consequences of branching in Verbascum thapsus (Scrophulariaceae). American Journal of Botany, 87, $1793-1796$.

Mabry CM, Wayne PW (1997). Defoliation of the annual herb Abutilon theophrasti: mechanisms underlying reproductive compensation. Oecologia, 111, 225-232.

Maschinski J, Whitham TG (1989). The continuum of plant responses to herbivory: the influence of plant association, nutrient availability and timing. American Naturalist, 134, 1-19.

Meyer GA (1998). Mechanisms promoting recovery from defoliation in goldenrod ( Solidago altissima) . Canadian Journal of Botany, $76,450-459$.

Obeso JR (1993). Does defoliation affect reproductive output in 
herbaceous perennials and woody plants in different ways? Functional Ecology, 7, 150-155.

Pate JS, Froend RH, Bowen BJ, Hansen A, Kuo J (1990). Seedling growth and storage characteristics of seeder and resprouter species of Mediterranean-type ecosystems of S. W. Australia. Annals of Botany, 65, 585-601.

Phillips IDJ (1975). Apical dominant. Annual Review of Plant Physiology, 26, $341-367$.

Reekie EG (1991). Cost of seed versus rhizome production in Agropyron repens. Canadian Journal of Botany, 69, 2678- 2683.

Riba M (1998). Effects of intensity and frequency of crown damage on resprouting of Erica arborea L. (Ericaceae). Acta Oecologica, $19,9-16$.

Sacchi CF, Connor EF (1999). Changes in reproduction and architecture in flowering dogwood, Cornus florida, after attack by the dogwood club gall, Resseliella clavula. Oikos, 86, 138 - 146.

Salemaa M, Vanha-Majamaa I, Gardner PJ (1999). Compensatory growth of two clonal dwarf shrubs, Arctostaphylos uva-ursi and Vaccinium uliginosum in a heavy metal polluted environment. Plant Ecology, 141, 79 - 91 .

Saruwatari MW, Davis SD (1989). Tissue water relations of three chaparral shrub species after wild-fire. Oecologia, 80, 303 308 .

Schier DA (1972). Apical dominance in multishoot cultures from aspen roots. Forest Science, 18, $147-149$.

Shi ZY (史竹叶), Liu WZ (刘文兆), Guo SL (郭胜利), Li FM (李凤民) (2003). Moisture properties in soil profiles and their relation to land form at Zhonglianchuan watershed. Agricultural
Research in the Arid Areas (干旱地区农业研究), 21 (4), 101 - 104. (in Chinese with English abstract)

Simons AM, Johnston MO (1999). The cost of compensation. American Naturalist, 153, 683 - 687 .

Sutherland S, Vickery RKJ (1988). Trade-offs between sexual and asexual reproduction in the genus Mimulus. Oecologia, 76, 330 -335 .

Trumble JT, Kolodney-Hirsh DM, Ting IP (1993) . Plant compensation for arthropod herbivory. Annual Review of Entomology, $38,93-119$.

van der Heyden F, Stock WD (1996). Nonstructural carbohydrate allocation following differences frequencies of simulated browsing in three semi-arid shrub. Oecologia, 102, 238-245.

Wang MB (王孟本), Li HJ (李洪建), Chai BF (柴宝峰) (1996) . Water ecophysiological characteristics of Caragana korshinskii. Acta Phytoecologica Sinica (植物生态学报), 20, 494 - 501. (in Chinese with English abstract)

Wang QZ (王全珍), Li QF (李青丰), Cui J(崔键), Wang YL (王月林), Bai RS (白日升), Dong ZF (董占峰) (2001). Path-coefficient analysis of seed yield with main agronomic charaters in Caragana korshinskii. Grassland of China (中国草 地), 23(3), 35-37. (in Chinese with English abstract)

Wellington AB (1984). Leaf water potentials, fire and the regeneration of mallee eucalypts in semi-arid, south-eastern Australia. Oecologia, 64, 360-362.

Westley LC (1993). The effect of inflorescence bud removal on tuber production in Helianthus tuberosus L. (Asteraceae). Ecology, 74, $2136-2144$. 